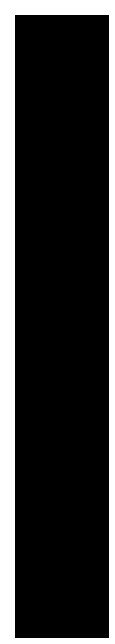

\title{
Update on the natural history of glaucoma
}

\begin{abstract}
Glaucoma is a progressive disease, and the rate of visual field (VF) progression is an important parameter that determines management decisions and is frequently used as an outcome measure in clinical trials. Different scoring systems have been used in the large randomized clinical studies, and have been found to vary in terms of sensitivity and specificity. All these systems measure change in VF from baseline, and the measurements are confounded by variability due to learning effect and test fatigue. For better patient management, the rate of change needs to be considered as well as the time to progression. Rate can be evaluated using trend analysis, as mean deviation (MD), clusters or points. Trend analysis suggests that the linear model is the 'best fit', at least for normal tension glaucoma. Using this model, it has been shown that the rate of progression in untreated normal-pressure glaucoma patients varies greatly despite similar ages and MD at baseline.

Eye (2007) 21, S2-S5; doi:10.1038/sj.eye.6702878
\end{abstract}

Keywords: glaucoma; visual fields trend analysis; event analysis

\section{Introduction}

Glaucoma is a progressive disease, and an understanding of its natural history of pathophysiological change is important in making management decisions, as well as in evaluating the effects of treatment. A number of recent randomized controlled clinical trials have

Moorfields Eye Hospital, London, UK

\section{Correspondence:}

R Hitchings, Moorfields Eye Hospital, London,

UK

Tel: +44207566 2818 .

E-mail: Roger.Hitchings@ moorfields.nhs.uk
R Hitchings

patient may not always present for the first time with VF defect measurements that reflect their pathophysiology. In addition, with repeated testing, they may perform better on one test than on the preceding one, even when the disease is progressively worsening. This is because of test learning and fatigue effects. Moreover, different methods to measure VF changes have different sensitivity and specificity. ${ }^{1}$

\section{Detection of disease progression by VF measurement}

The main scoring systems used in VF analysis studies may be referred to as the Advanced Glaucoma Intervention Study (AGIS), ${ }^{2}$

Collaborative Initial Glaucoma Treatment Study (CIGTS), ${ }^{3}$ and Glaucoma Change Probability (GCP) methods, all of which are event-based analyses, and pointwise linear regression (PLR), which is a trend-based approach. For event-based analysis, the criterion for progression (the event) is defined at the start of the study, and progression is confirmed when VF changes fall below a pre-set threshold, compared with baseline. All the eventbased methods measure VF change from baseline, with only two test points compared at any one time. Trend-based analyses consider changes over time using several VF measurements, and therefore are more sensitive at detecting progression and the rate of change.

Vesti et $a l^{4}$ compared the four main scoring systems in a study of 76 patients with progressive open-angle glaucoma. VFs were measured twice a year for 7 years to see how the individual methods picked up computergenerated fluctuations; the three conditions tested were high, moderate, and no variability. Time to detection of disease progression and method specificity were the main outcome measures. Figure 1 shows that GCP was very good at demonstrating and identifying change early on, but its ability to do so was affected by the degree of fluctuation more than for any other method. PLR was very specific at 
identifying changes in VF, but had the longest times to confirmed progression. The other two methods had the highest specificity and classified fewer cases of progression than the other methods.

Both event and trend analyses were used in the Collaborative Normal Tension Glaucoma (CNTG) study, ${ }^{3}$ in which treated and untreated patients with normaltension glaucoma (NTG) were compared. This study considered, through event analysis, how many months of follow-up it took before progression was detected. The criterion for progression was the presence of two or more VF points depressed by $\geqslant 10 \mathrm{db}$ from baseline, on average, confirmed in four of five consecutive VF tests. The scatter plot for those patients who were untreated shows, over time, using mean deviation (MD) in the total measure of change, the large inter-patient differences in the time to detection of progression. The trend is indicated by the slope of the change in the MD. ${ }^{5}$

In the Early Manifest Glaucoma Trial (EMGT), ${ }^{5}$ event analysis alone was used. Again, treated and untreated patients with early glaucoma were compared. CGP maps were plotted; the threshold value of each test point location in every follow-up field was mapped to an average of the threshold values from the same test point in two baseline fields. Progression was said to have occurred when three VF points showed change on three successive VF tests. ${ }^{5}$

\section{PLR analysis}

In trend analysis, three different types of statistical method (exemplified by spatial processing, temporal modulation, and the three-omitting method) may be used to improve measurement and distinguish

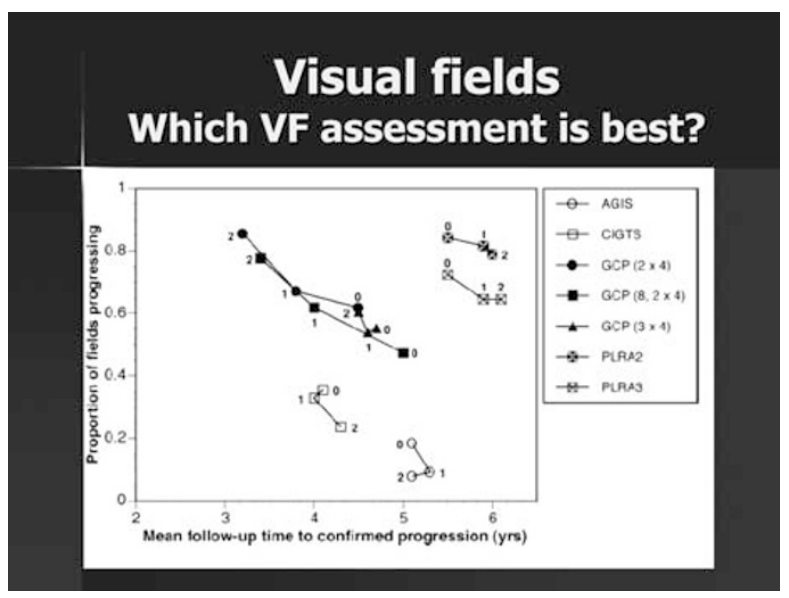

Figure 1 Comparison of the four visual field scoring systems in detecting progression. (Reproduced from Vesti et al ${ }^{4}$ with permission of Invest Ophthalmol Vis Sci via Copyright Clearance Center.) variability from progression. A plot of specificity against proportion of fields progressing shows that these methods, used with PLR, are able to improve specificity by demonstrating reduced sensitivity changes with time. Spatial processing shows an apparent improved ability to detect change through the removal of some of the fluctuation (or 'noise') that occurs. Temporal modulation, an alternative method, uses the moving average technique on two sequential point sensitivities. The three-omitting method aims for sustainability in repeat testing. ${ }^{6}$ With this approach, a point is flagged as progressing if it shows a significant negative slope of at least $-1 \mathrm{~dB} /$ year at the $1 \%$ significance level (point $n=1)$, and the slopes obtained by using points 1 to $(n-1)$ and either point $(n+1)$ or point $(n+2)$ both also satisfy this same criterion. The logic of this approach is that the $n-1$ point may be lower than point $n$ by chance, and so if it is missed out, there will be a more conservative estimate of progression. This approach, therefore results in fewer false-positive results, but is still sensitive to progression (Figure 2).

A study conducted at Moorfields Eye Hospital looked specifically at the time (in months) for untreated NTG patients to show VF change. ${ }^{7}$ The records from the database were analysed using PLR with the threeomitting method (criteria as above) to identify VF change. The first two VFs were omitted to allow for test learning effects, so that the third was deemed the baseline, and the three-omitting method was applied to correct for test fatigue. The longer-term follow-up data are now available and show that, using a low significance level of $5 \%$ for 476 right eyes and 479 left eyes, over $40 \%$ of the eyes could be said to have progressed at some stage. Increasing the significance level to $1 \%$ led to a fall

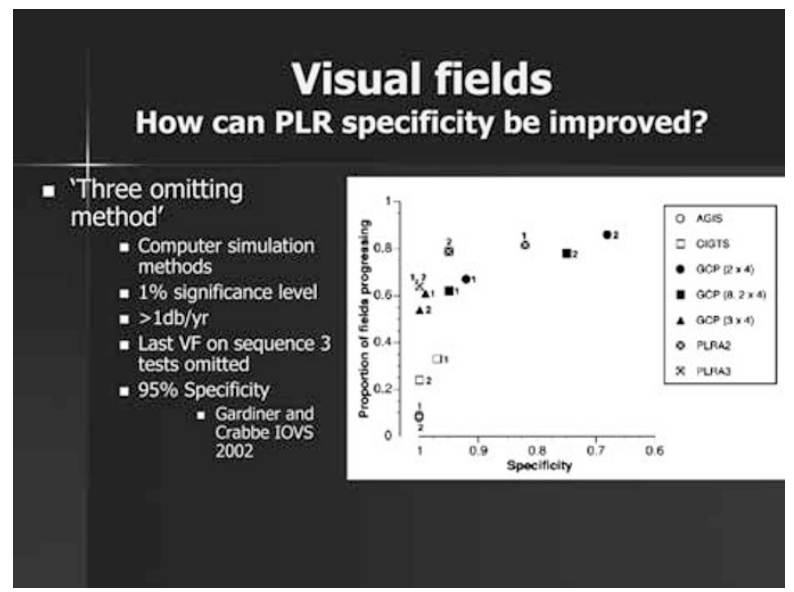

Figure 2 Improvement of specificity of pointwise linear regression analysis with the three-omitting approach. (Reproduced from Vesti et $a l^{4}$ with permission of Invest Ophthalmol Vis Sci via Copyright Clearance Center.) 


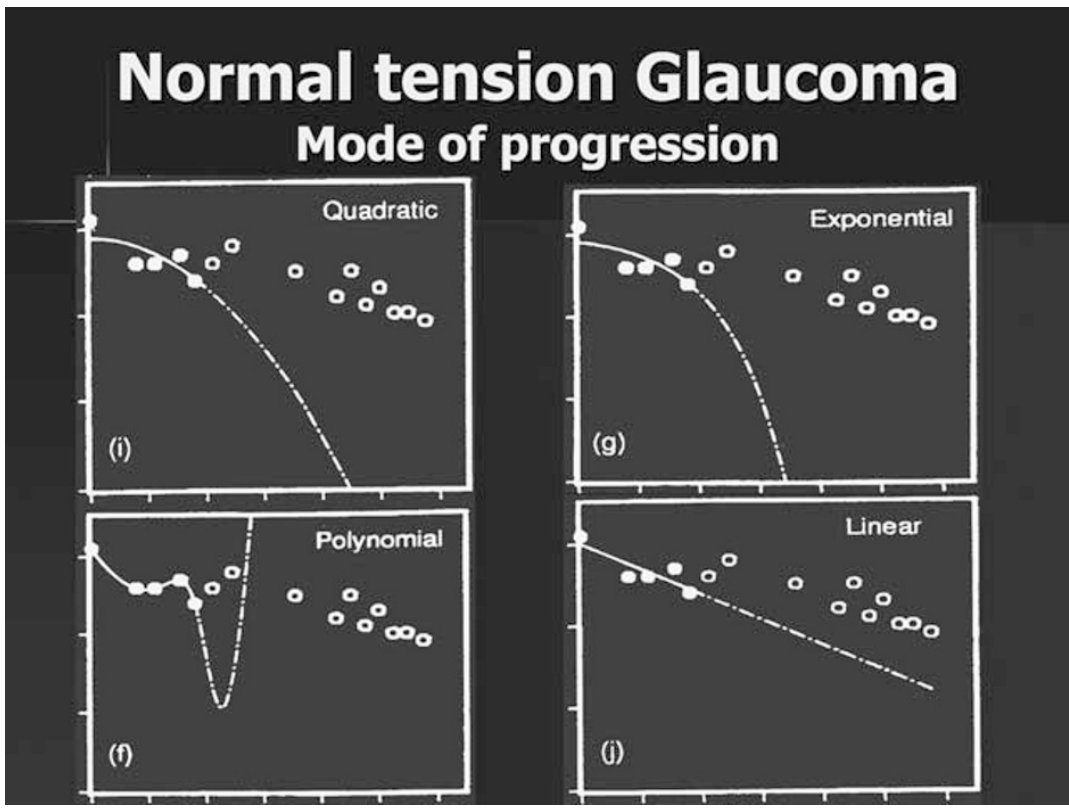

Figure 3 Analysis of different modes of progression showing that linear progression provides the best 'fit'.

in numbers who were defined as progressing to about one-quarter (26-27\%) over the follow-up period of at least 5 years. Progression was detected at between three and 38 visits (average of 17 visits). The minimum time from baseline to progression was 4 months, and the average time was 6 years. The rate of loss ( $\mathrm{dB} /$ year) varied greatly, with an average of under $3 \mathrm{~dB}$.

\section{Phenotypic characteristics}

Data from the Moorfields study thus show that the rate of progression varies greatly among glaucoma patients without treatment. For this reason, the phenotypic characteristics of the eyes were examined to see if there was a pattern by evaluating the severity of the disease and age at diagnosis. For each phenotypic variable, the group was divided into two, based on the uppermost (slowest) and lowest (fastest) quartiles for progression. Patient ages ranged from 40 to 80 years, and showed no correlation with disease severity (expressed as MD). Similarly, age did not correlate with rate of progression. In these NTG patients, it was not possible to determine any significant phenotypic differences between the slow- and fast-progressing eyes.

\section{Conclusion}

In conclusion, event and trend analysis are both suitable for natural history studies of the progression of glaucoma, while the AGIS and CIGTS scoring systems are poor at picking up cases of progression. Trend analysis suggests that the linear model is the best 'fit', at least for NTG (Figure 3). ${ }^{8}$ Using the linear model, it has been shown that the rate of progression in untreated NTG patients varies greatly despite similar ages and MD at baseline.

\section{References}

1 AGIS Investigators. The Advanced Glaucoma Intervention Study (AGIS): 7. The relationship between control of intra-ocular pressure and visual field deterioration. Am J Ophthalmol 2000; 130: 429-440.

2 Lichter PR, Musch DC, Gillespie BW, Guire KE, Janz NK, Wren PA, et al., CIGTS Study Group. Interim clinical outcomes in the Collaborative Initial Glaucoma Treatment Study comparing initial treatment randomised to medications or surgery. Ophthalmology 2001; 108: 1943-1953.

3 Flammer J. Fluctuations in the visual field. In: Drance SM, Anderson, DR (eds). Automatic Perimetry in Glaucoma a Practical Guide. Grune and Stratton Inc.: Orlando, FL, USA, 1985, pp 161-173.

4 Vesti E, Johnson CA, Chauhan BC. Comparison of different methods for detecting glaucomatous visual field progression. Invest Ophthalmol Vis Sci 2003; 44: 3873-3879.

5 Heijl A, Leske MC, Bengtsson B, Bengtsson B, Hussein M, Early Manifest Glaucoma Trial Group. Measuring visual field progression in the Early Manifest Glaucoma Trial. Acta Ophthalmol Scand 2003; 81: 286-293.

6 Gardiner SK, Crabb DP. Examination of different pointwise linear regression methods for determining visual field progression. Invest Ophthalmol Vis Sci 2002; 43(5): 1400-1407. 
7 Membrey WL, Poinoosawmy DP, Bunce C, Fitzke FW, Hitchings RA. Comparison of visual field progression in patients with normal pressure glaucoma between eyes with and without visual field loss that threatens fixation. $\mathrm{Br} \mathrm{J}$ Ophthalmol 2000; 84(10): 1154-1158.
8 McNaught AI, Crabb DP, Fitzke FW, Hitchings RA. Modelling series of visual fields to detect progression in normal-tension glaucoma. Graefes Arch Clin Exp Ophthalmol 1995; 233(12): 750-755. 